\title{
EFEITOS DE SOLUÇAO HIPERTONICA POR VIA ENDOVENOSA NOS ESPAÇOS PERIVASCULARES CEREBRAIS DO CÃO
}

\author{
PAOLO CONTU* \\ MATILDE ACHAVAL ELENA**
}

Os mecanismos anátomo-funcionais dos espaços perivasculares do sistemá nervoso central (SNC) constituem assuntos muito discutidos. No presente trabalho apresentamos os resultados obtidos no estudo da membrana pio-glial, vaso e espaço perivascular após injeções hipertonicas endovenosas no cão, parcialmente comunicados em nota prévia (Contu, 1964) $)^{5}$ e que agora serão analisados à luz de recentes conhecimentos sobre o assunto.

Em toda superfície do SNC o dispositivo vascular se ramifica em ramos arteriais e venosos de até 100 micra de diâmetro, que penetram no interior do parênquima nervoso, diminuindo de calibre e separados do mesmo parênquima pelos espaços perivasculares de Virchow-Robin. Estes espaços segundo as observaçōes de Virchow ${ }^{10}$, Robin ${ }^{16}$, His ${ }^{9}$, Weed ${ }^{20}$, Schaltenbrand \& Bailey ${ }^{17}$, Gadrat ${ }^{6}$, Patek 14, Kuhlembeck ${ }^{10}$, Bairati ${ }^{2}$, Glees ${ }^{8}$, Woollam \& Millen ${ }^{22}$, Bairati ${ }^{3}$, Tschirg ${ }^{18}$, entre outros, são atualmente bem conhecidos como continuação do espaço subaracnoidal. A nível capilar a neuroglia substitui a piaaracnóide, desaparecendo assim o espaço perivascular e observando-se intimo contato dos pés sugadores com a parede capilar mesma, base morfológica da barreira sangue-cérebro.

\section{MATERIAL E METODOS}

Utilizamos a área do espaço paraolfatório basal (correspondente a substancia per(urada anterior humana) de 16 cães de raças diferentes discriminados na tabela 1 , pelo número, sexo, peso e idade.

As modalidades técnicas foram as seguintes:

Nos cães C1-C24, em anestesia total nembutálica (33mg por $\mathrm{Kg}$ de peso) foram injetados na veía femural $20 \mathrm{cc}$ de uma solução hipertônica de cloreto de sódio (20 por mil) muito lentamente, levando de 6 a 10 minutos. Contemporaneamente na reglăo temporal esquerda, cortanło com incisão longitudinal a pele, o músculo temporal e - perióstio, foi praticada a trepanaçáo de uma área óssea de $2-3 \mathrm{~cm}$ de diametro,

Trabalho realizado no Laboratório de Neuroanatomia do Departamento de Ciências Morfológicas do Instituto de Biociências da UFRGS, com auxilio do C.N.Pq. e da UFRGS: - Chefe do Laboratório de Neuroanatomia e Pesquisador IA do C.N.Pq; * Professor visitante do Curso de Pós-graduacăo em Neuroanatomia. 


\begin{tabular}{|c|c|c|c|c|}
\hline & CATO Ne & SEXO & PESO & IDADE \\
\hline & C1 & o & $13 \mathrm{Kg}$ & adulto \\
\hline & $C 7$ & $\sigma$ & $21 \mathrm{Kg}$ & adulto \\
\hline & C8 & $\sigma^{\circ}$ & $13 \mathrm{Kg}$ & adulto \\
\hline$\underline{E}$ & C10 & 9 & $17 \quad \mathrm{Kg}$ & adulto \\
\hline$\sum_{i=1}^{\infty}$ & C11 & 0 & $19 \mathrm{Kg}$ & adulto \\
\hline $\mid \begin{array}{l}\mathbf{b} \\
a \\
x\end{array}$ & $C 12$ & q & $15 \quad \mathrm{Kg}$ & adulto \\
\hline n & $C 15$ & 0 & $9 \quad \mathrm{Kg}$ & adulto \\
\hline 0 & $C 20$ & م & $9,5 \mathrm{~kg}$ & adulto \\
\hline & C23 & $\sigma^{2}$ & $11 \quad \mathrm{Kg}$ & adulto \\
\hline & $C 24$ & 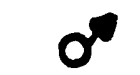 & $13 \mathrm{Kg}$ & adulto \\
\hline & $C 25$ & q & $9 \quad \mathrm{~kg}$ & jovem \\
\hline لّ & $C 26$ & $\delta$ & $18 \mathrm{Kg}$ & adulto \\
\hline$\frac{x}{z}$ & $C 27$ & & $9.5 \mathrm{Kg}$ & adulto \\
\hline $\begin{array}{l}0 \\
n\end{array}$ & 529 & $\delta$ & $8,5 \mathrm{~kg}$ & adulto \\
\hline n & $C 29$ & 9 & $8 \quad \mathrm{Kg}$ & jovem \\
\hline & 030 & & $9 \mathrm{Kg}$ & jovem \\
\hline
\end{tabular}

Tabela 1-Relapato dos casos experimentals o casos controles discriminados por cóligo, sexo, peso 6 idade.

introduzindo, após incisâo da dura-mater, no espaço subaracnoidal de um sulco cerebral, um pequeno tubo de polietileno ligaco a uma seringa com $20 \mathrm{cc}$ de uma soluçáo a $1 \%$ de tinta da India, injetada lentamente durante 6-8 minutos; 20 minutos apos, procedia-se à fixaça em vío do animal com uma soluçáo de formol a $10 \%$. Os câes C23-C30 usados como controle, foram só fixados em vivo, em anestesia nembutálíca.

Procedia-se, em seguida, a extraçáo do encéfalo que fol colocado em bandeja com a base para cima e, após a abertura do cavo subaracnoidal, foi delimitado com corte em profundidade até a área estriada o espaço paraolfatório que era retirado e fixado em formol a $10 \%$ e liquido de Rio-Hortega e inclufdo em parafina.

Para cada caso foram cortados grupos de secçóes seriadas de espessura de 20 micra, Indo de auperticle até $10 \mathrm{~mm}$ de protundidade, corados com o método de Mallory. 
Com a camara lúcida eram executados desenhos com o aumento de 700 diametros, limitados a uma superficie quadrada de $7 \mathrm{~cm}$ de lado, desenhando-se nesta área os vasos e a membrana pio-glial: para cada caso foram executados 10 desenhos, correspondentes cada a $1 \mathrm{~mm}$ sucessivo de profundidade (Figs. 1 e 2).

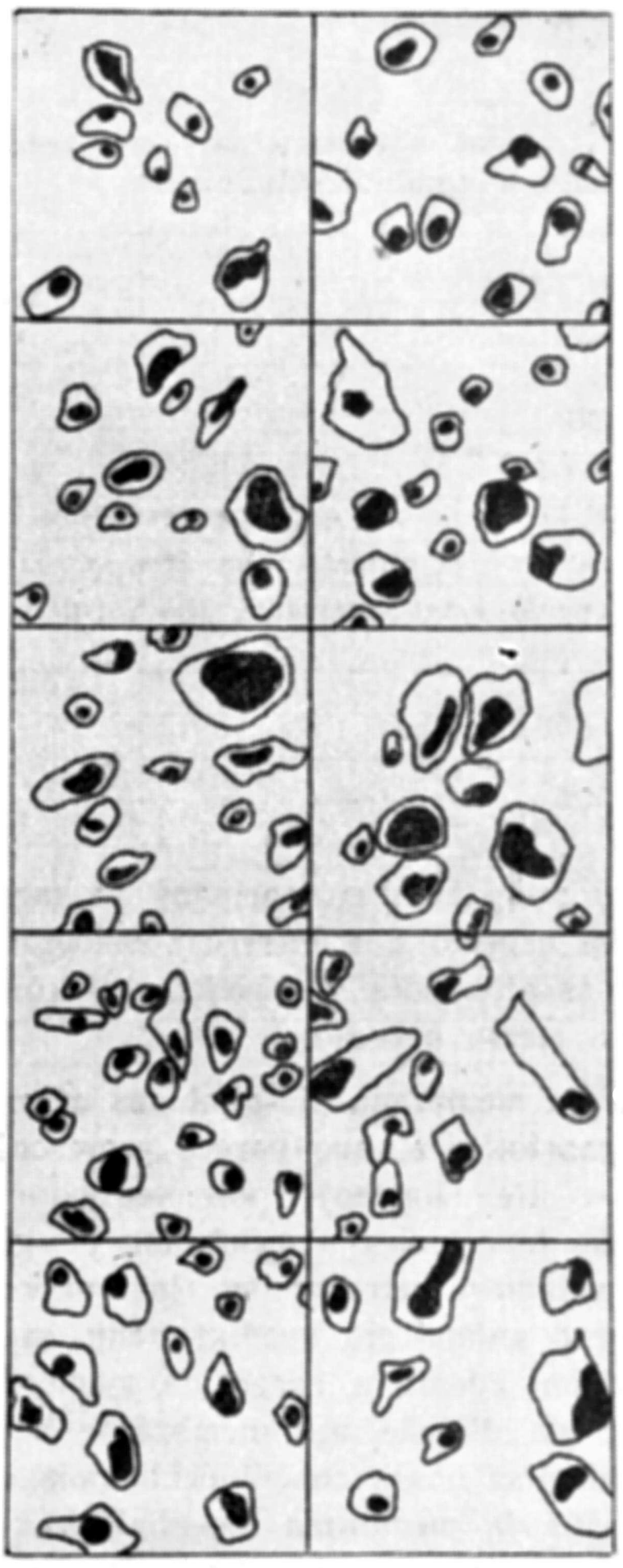

Fig. 1-Caso 24, experimental: fotografia dos 10 desenhos feitos com a camara lúcida. evidente o au. mento dos espacos perivaxculares por retraça da membrana pio. glial.

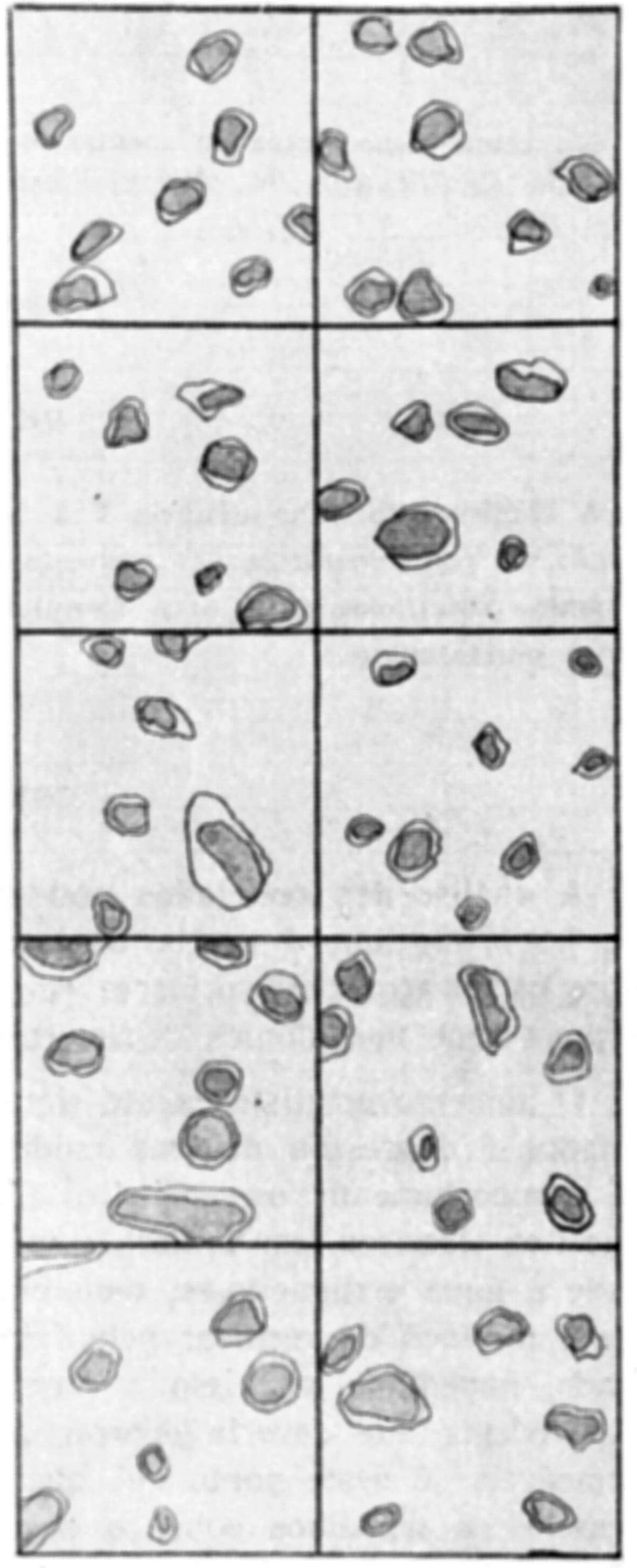

Fig: z-Caso 26, controle: fotografia dos 10 desenhos feitos com a camarc luicida. Comparando com a fig anterior é bem visivel como apa. recem 08 espacos perivasculares normalmente. 
Cada desenho era depois estudado separadamente calculando em $\mathrm{mm}$ com o planimetro a área dos vasos e a área da membrana pio-glial, cuja diferença dá a áreła dos espaços perivasculares. Na tabela 2 e no histograma 1 estão representados os dados obtidos neste trabalho. Para o cálculo do erro-padrão da média (e) foi empregada a seguinte fórmula:

$$
y=-\frac{s(x-\bar{x})^{2}}{n(n-1)}
$$

Na comparação entre as médias de grupos tratados e controle foi empregado o teste de significancia de Student-Fischer mediante o seguinte cálculo:

$$
t=\frac{\bar{x}}{-1}-\frac{x_{2}}{1}
$$

\section{RESULTADOS}

A análise estatística (Tabela 2 e histograma 1) revela no grupo experimental em relação ao grupo controle: a) aumento estatisticamente significativo na área pio-glial; b) pouca significancia na área vascular; c) aumento estatisticamente significativo do espaço perivascular.

\section{COMENTARIOS}

A análise dos resultados obtidos e que estão bem evidenciados na tabela 2 e no histograma 1 revela de imediato um aspecto que merece consideração sobre os espaços perivasculares em relação às atividades que podem ser consideradas base morfológica de intercâmbio no tecido nervoso.

$\mathrm{O}$ aumento estatisticamente significativo na membrana pio-glial dos animais tratados é expressão de uma modificação morfológica, que parece representar um comportamento osmométrico (Gimeno e De Gimeno) ${ }^{7}$ pois segundo os conceitos clássicos, em contato com a solução hipertonicả a membrana pio-glial perde a água errugando-se, diminuindo de volume e retraindo-se. Isto naturalmente foi fácil de verificar pelo fato de que o animal era imediatamente sacrificado, impedindo por isso a formação de um edema a cargo da membrana glial, edema que deveria ocorrer pelas leis da difusão nas membranas semipermeáveis: é neste ponto que queremos salientar nossa contribuição, pois são escassos os trabalhos sobre o comportamento da membrana pio-glial, a qual assim poderá ser analizada mais cuidadosamente no conjunto das barreiras no tecido nervoso. Estas barreiras não podem ser esquecidas por causa da predominância da barreira hemato-encefálica que com as características morfológicas descritas no distrito capilar é base para interpretar fisiologicamente a seletividade no intercâmbio (Brierley ${ }^{4}$, Reese \& Karnowsky ${ }^{15}$, Weestergaard \& Brightman ${ }^{21}$, Oldendorf ${ }^{12}$ ). Merecem atenção também a barreira meníngea a nível aracnóideo (Nabeshima, Reese, Landis \& Brightman) ${ }^{11}$, a barreira hematoneural a nivel do perineuro e capilares endoneurais (Olsson \& Reese ${ }^{13}$, Aker ${ }^{1}$ ). 


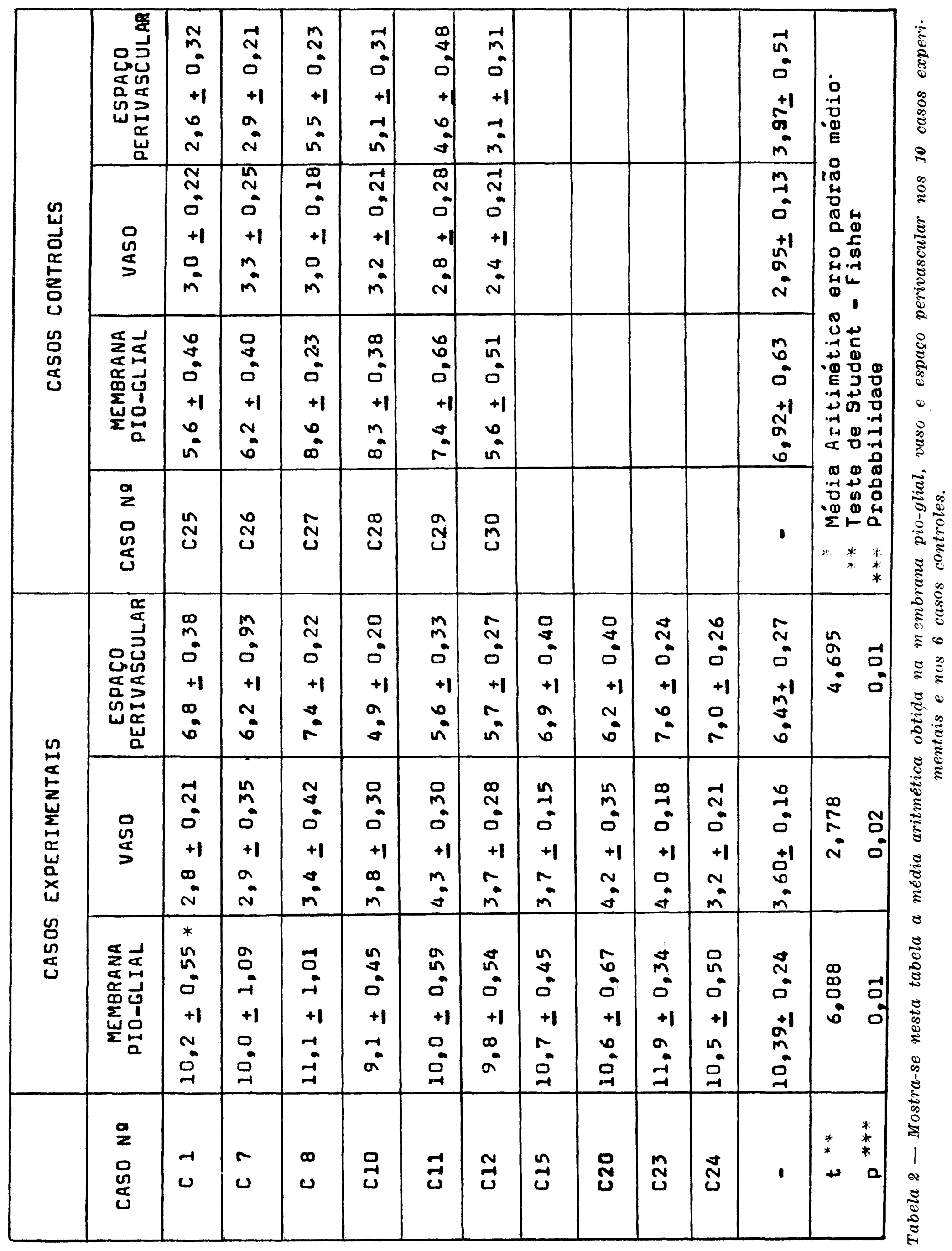




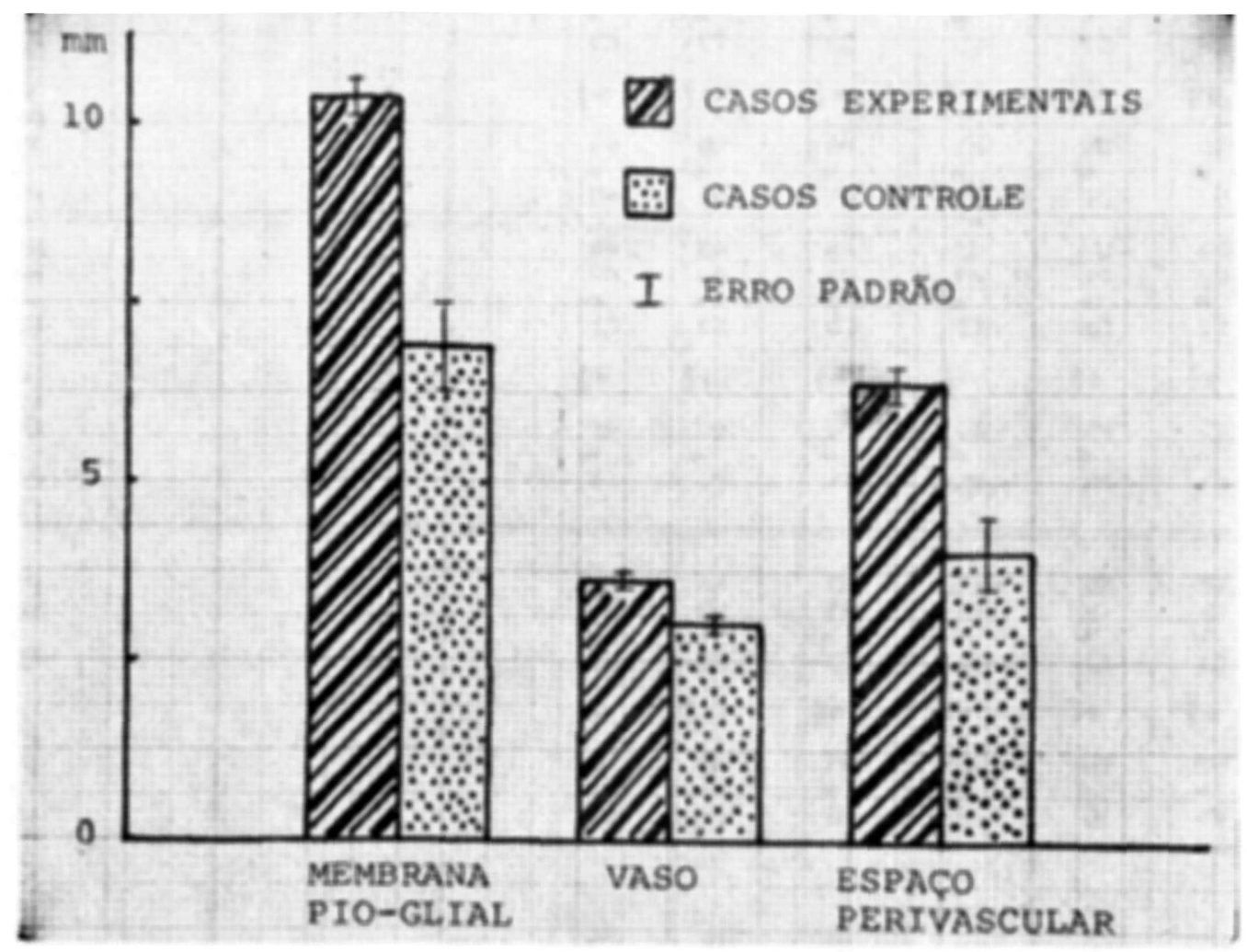

Histograma 1 - Evidencia-se o aumento do espaco perivascular,
devido ao aumerato significativo da membrana
pio-glial, o que no acontece no vaso.

Nossos resultados podem ser resumidos no aumento do espaço perivascular em detrimento da alteração da membrana pio-glial, sem dar importåncia à área vascular que é estatisticamente insignificante. Consequentemente parece-nos que a membrana pio-glial dos espaços perivasculares não possa ser considerada mua estrutura estática em vista de resposta à solução hipertônica, o que nos leva a considerá-la como parte integrante do complexo neurônio-glia-meninge.

\section{RESUMO}

Os autores estudam o comportamento dos vasos do espaço paraolfatório basal penetrando no parênquima nervoso, após injeção de solução hipertônica de cloreto de sódio a 20/1000, por via intravenosa e de tinta da fndia a $1 \%$ por via subaracnoidal em 10 cães de raças diferentes e mantendo outros 6 como controle. $\mathrm{O}$ estudo foi realizado utilizando o método de Mallory no material que era cortado em seç̧ões de 20 micra seriadas, indo da superfície até $10 \mathrm{~mm}$ de profundidade executando com a câmara lúcida 10 desenhos dos vasos e da membrana pio-glial em cada caso. Cada desenho era depois estudado separadamente calculando em $\mathrm{mm}$ com o planímetro a área dos vasos e a área da membrana pio-glial. A análise estatistica revelou um aumento significativo do espaço perivascular pela ação da solução hipertônica, o que parece ser expressão do comportamento osmométrico da membrana pio-glial, que assim não pode ser considerada como estrutura estática mas parte integrante no complexo neurônio-glia-meninge. 


\section{SUMMARY}

Effects of endovenous hipertonic solution in the cerebral perivascular spaces in dogs.

The authors studied the behavior of vessels penetring the nervous parenquima in the basal paraolfatory space after an intravenous injection of the lipertunic sodium chıorice solution $(20 / 1000)$, plus a $1 \%$ India ink solution injected via subarocnoical space. The experiments were performed in 10 dogs of different races keeping 6 as controls.

The experiment followed Mallory's method and consisted in cutting series of 20 m.crons sections, going from tine surrace down to 10mm deep, 10 plates (in eacn case) of tine vessels and the pio-glial membrane were drawn up with tne and of a "camera lucida". The statist.cal analysis showed a significant enhance in the perivascular space due to the hipertonic solution which seems to results from an osmotic phenomenon through the pio-glial membrane. In this way, the pio-glial membrane can be considered as an active structure in the neu onal-ghal-meninge complex.

\section{REFERENCIAS}

1. AKER, D. - A study of hematic barrier in peripheral nerves of albino rabbits. Anat. Rec. 174:21, 1972.

2. BAIRATI, A. - Problemi vecchi e nuovi della glia S:stema Nervoso (Milano) $1: 1-4,1950$.

3. BAIRATI, A. - Perivascular relationship of the neuroglia cells. In Biology of Neuroglia - W. Windle ed. Charles C. Thomas, Springfield (Illinois), 1958, pag. 85-98.

4. BRIERLEY, J. B. - The blood brain barrier: structural aspects. In Metabolism of the Nervous System, - D. Richter ed., New York, Pergamon Press, 1957, pag. 121-135.

5. CONTU, P. - Observações exper:mentals sobre os espaços perivasculares no căo (nota prévia). Arq. Inst. Anat. Porto Alegre 6:5-20, 1963-64.

6. GADRAT, J. - De l'Espace Perivasculaire du Cerveau et de la Moelle. Librairie Ballière et Fils, Paris, 1931.

7. GIMENO, A. L. \& DE GIMENo, M. A. F. - Nociones de Fisiologia Celular. Editorial Universitária, Buenos Aires, 1963.

8. GLEES, P. - Neuroglia: Morphology and Function. Charles C. Thomas, Sprinfield (Illinois), 1953.

9. HIS, W. - Ueber ein perivascularen Canalsystem in der Centralorganen und ueber Clessen Beziehung zum Lymphsystem. Zeitsch. f. Wiss. Zool., $15: 127,1865$.

10. KUHLEMBECK, H. - Virchow-Rob:n spaces, spaces of His-Held and the:r relation to the membrana limitans perivascularis. Anat. Rec., 109:375, 1951. 
11. NABESHIMA, S.; REESE, T. S.; LANDIS, D. M. D. \& BRIGHTMAN, M. W. Junctions in the meninges and marginal glia. J. Comp. Neur. 164:127, 1975.

12. OLDENDORF, W. H. - The Blood-Brain Barrier. Exp. Eye Res. Suppl. 177-19u. 1977.

13. OLSSON, $Y$ REESE, T. S. - Permeability of vasa nervorum and perineurium in mouse sciatic nerve studied by fluorescence and electron microscopy. J. Neuropath. Exper. Neurol. 30: 105, 1971.

14. PATEK, P. R. - The perivascular spaces of the mammalian brain. Anat. Rec. 88:124, 1944.

15. REESE, T. S. \& KARNOVSKY, M. J. - Fine structural localization of bloodbrain barrier to exogenous peroxidase. J. Cell. Biol. 34:207, 1967.

16. ROBIN, C. - Recherches sur quelques particularités de la struture des capillaires de l'encephale. Journal de la Physiologie Des Hommes et des Animaux. 2:537, 1850.

17. SCHALTENBRAND, G. \& BAILEY, P. - Die perivasculare Pia-gliamembran des Gehirns. J. Psychol. Neur., 35:199, 1928.

18. TSCHIRG, R. D. - The Blood-brain Barrier. In Biology of Neuroglia, W. Windle ed., Charles C. Thomas. Springfield (Illinois), 1958, pág. 130-138.

19. VIRCHOW, R. - Ueber die Erweterung kleinerer Gefaesse. Arch. $f$. Pathol. Anat. 3:427, 1851 .

20. WEED, L. A.- An anatomical consideration of the cerebrospinal fluid. Anat. Rec. $12: 461,1917$.

21. WESTERGAARD, E. \& BRIGHTMAN, M. W. - Transport of proteins across normal cerebral arterioles. J. Comp. Neunol. 152:17, 1973.

23. WOOLLAM, D. H. \& MILLEM, J. W. - The perivascular spaces of the mammalian central nervous system and their relation to the perineuronal and subaracnotd spaces. J. Anat. (London), $89: 193,1955$.

Laboratorio de Neuroanatomia, Departamento de Ciencias Morfologicas do Instituto de Biociencias da UFRGS - Rua Barmento Leite 8/n. - 90000 - Porto Aleare, R8 - Brasil. 